\title{
Die geographische Verbreitung der Schweizen
}

\begin{abstract}
Allein im Gebiet des Baltischen Landrückens finden sich dreizehn, im weiteren Bereich von Rhein und Weser über zwei Dutzend "Schweizen". Mit dieser etwas ungewohnten Mehrzahlform ( Einzahl : Die Schweiz) bezeichnen wir jene Orts - und Gebietsnamen, welche aus irgend einem Grunde "Schweiz" enthalten. Auch in aussereuropäischen, überseeischen Ländern finden sich viele Beispiele. Die Deutung und Erklärung dieser Namengebung stellt interessante Probleme, auf die im folgenden eingegangen werden soll.
\end{abstract}

Aus geographischer Sicht hat schon Alfred Hettner über eine der "Schweizen" (Elbsandsteingebirge)(1) eine grössere Abhandlung geschrieben; Irmfried Siedentop veröffentlichte vorläufige, diesen Beitrag vorbereitende $\mathrm{Zu}$ sammenfassungen der ihm bekannten "Schweizen"(2). Die gegenwärtige Arbeit beabsichtigt, eine möglichst vollständige Zusammenstellung der "Schweizen" zu erreichen; ohne die dan kenswerte Mithilfe vieler Geographen, einiger Journalisten, Privatpersonen und Behörden wäre sie wiederum ein Torso geblieben.

Der Begriff "Schweizen" könnte mit wenigen Worten dahingehend definiert werden, dass man darunter die Gesam theit der zumeist mit idealen Landschaftselementen ausgestatteten und mit der zusätzlichen Bezeichnung "Schweiz" versehenen Oertlichkeiten und Regionen versteht; um ein Beispiel zu nennen: La Suisse Normande. Eigentlich erst recht spät, ausgangs des 18. und im 19. Jahrhundert lernte man die abwechslungsreichen landschaftlichen Schönheiten der Schweiz schätzen; Goethes Schweizer Reisen mögen für viele dazu ein Ansporn gewesen sein. Die Entwicklung im Verkehrswesen ermöglichte es vielen Reisenden, die schweizerischen Gebiete kennenzulernen und darüber zu berichten; das hatte sehr viele Schweiz-Benennungen zur Folge.

Das genaue Namengebungs-Jahr für die verschiedenen "Schweizen" anzugeben, ist meist nicht möglich. Das Elbsandsteingebirge wurde beispielsweise von dem im Jahre 1766 nach Dresden berufenen Schweizer Maler Anton
Graff als Sächsische Schweiz bezeichnet (3). Im Laufe der folgenden Jahrzehnte wurden danr auch die Fränkische und die Holsteinische Schweiz zu feststehenden Begriffen. Bis sich jedoch die neuen Bezeichnungen durchsetzten, verstrich eine geraume Zeit; das galt auch für die Aufnahme in die Literatur. Nur die bekann. teren "Schweizen" findet man in Atlanten, Hand- und Lehrbüchern sowie in den Nachschlagewerken.

Die Frage nach der genauen Anzahl von "Schwe zen" in aller Herren Länder ist zwar durchaus berechtigt, lässt sich jedoch nicht präzise beantworten, da in die Wege geleitete Nachforschungen bei einer Reihe von vermeintlichen "Schweizen" leider nicht zum Ziele führten. Doch darf man wohl mit rund hundert "Schweizen" rechnen.

Ebenfalls berechtigt ist die Frage, warum denr eigentlich nicht für andere, auch mit landschaftlichen Superlativen versehene Länder, "Na chprägungen" analog den "Schweizen" vor genommen worden sind. Sie ist nicht allein damit zu beantworten, dass die Schweiz anderen Staaten einfach den Rang abgelaufen hat; vielmehr werden im Einzelfall auch manche Zufälligkeiten mit im Spiel gewesen sein. Freilich ist überraschend, dass die Alpen oder nicht-schweizerische Gebiete nur in wenigen Fällen zu Namengeburgen herangezogen wur den. Beispiele sind: Japanische-, AustralischeNeuseeländische-, Béler Kalk(Hohe Tatra) und Fünens Alpen (mit $128 \mathrm{~m}$ Höhe in Dänemark); Ruanda: das Allgäu Afrikas; Osaka: das japanische Salzburg (4); Neuseeland:das Grossbritannien der Südsee; Shillong (Khasigebirge): Scotland of the East (5).

Worüber man immer wieder erstaunt sein könnte, ist die Tatsache, dass die "Schweizen" überwiegend gar keine oder doch nur geringfügige Aehnlichkeiten mit der wechselvollen Lands chaftsgestaltung des "sprachlichen Mutterlandes". besitzen. Aber darauf kam es

Dr. I. Siedentop, Wilhelmshöhe 17

D - 2420 Eutin 
im Grunde genommen auch gar nicht an, als man die Namengebung vornahm. Man wollte fern vom Alpenstaat, der eben der Inbegriff des landschaftlich Schönen und Ausdruckvollen (6) geworden war, seine eigene "Schweiz" vor Augen haben; durch die "Nachprägung" für bevorzugte Landschaftsräume sollte dies dokumentiert werden. Dass im Bereich des Baltischen Landrückens mit seinen lieblichen Seen und beachtlichen Hügeln, mit seinen meistens gepflegten Waldungen und gern aufgesuchten Ortschaften eine ganze Reihe von "Schweizen" anzutreffen ist, kann so gesehen wohl doch nicht so ganz reiner Zufall sein.

Ueber die Hälfte der bekannten "Schweizen" ist im nördlichen Mitteleuropa etwa zwischen dem Baltikum und den Beneluxländern anzutreffen; aus diesem Raum stammten bereits zur "Gründerzeit" sehr viele Besucher der Schweiz. Es finden sich hier Ballungen von "Schweizen" und Vorkommen mit grossen Zwischenräumen, ohne dass ein systematisches Verteilungsmuster zu erkennen wäre; in Mittel hessen und im Thüringer Becken fehlen "Schweizen" überhaupt.

Um nun die einzelnen "Schweizen", für die man die Lage zwar exakt anzugeben vermag, deren Begrenzung allerdings zumeist fliessend ist, zu interpretieren, wurde aus Gründen der schnelleren Auffindbarkeit die alphabetische Reihenfolge gewählt, wobei bei der Kurzbeschreibung die Gefahr der Wiederholung besteht; aber dieser Schönheitsfehler wird durch die bessere Uebersichtlichkeit gemildert. Die Länge der einzelnen Abschnitte darf nicht in Beziehung zur Bedeutung der jeweiligen "Schweizen" gesetzt werden, war doch das erreichbare Material leider von recht unterschiedlichem Umfange. Die "Schweizen" verdanken ihre Bezeichnung und ihr Ueberleben uberwiegend landschafts-ästhetischen Gesichts punkten, die darum auch entsprechend zu berücksichtigen sind.

Bevor mit der Interpretation dęr einzelnen "Schweizen" begonnen werden soll, muss daraufhingewiesen werden, dass leider nicht für sämtliche Regionen trotz z. T. mehrfacher An- fragen,entsprechende Unterlagen zur Verfügung gestellt wurden.

Einige Aehnlichkeiten mit "The Switzerland of America" im US Bundesstaat Oregon besitzen die Schweizer Voralpenlandschaften. Einer Aufnahme(7), welche den dortigen Landschafts charakter vor Augen führt, ist zu entnehmen, dass ein sich in einem See widerspiegelnder Berg ganz auffallend dem Niesen im Berner Oberland gleicht.

Die Argentinische Schweiz umfasst etwa den mittleren Teil des Patagonischen Seengebietes am Ostabfall der südlichen Anden in der Provinz Rio Negro und weist in landschaftlicher Beziehung viele Uebereinstimmungen $m$ it der Schweiz auf (8); hier liegt in einer Gegend von einmaliger Schönheit das bedeutende Turistikzentrum San Carlos de Bariloche, ein von ansehnlichen, mit Gletschern bedeckten Berggipfeln (z. T. Grossglockner- Höhe erreichend) und tausendjährigen Waldungen umgebener Ort. Flüsse, Wasserfälle und Seen mit darin befindlichen Inseln beleben das Landschaftsbild. Bemerkenswert ist der See Nahuel Huapi, 530 qkm (Genfer See $581 \mathrm{qkm}$ ), der lebhaften Schiffsund Bootsverkehr aufweist und durch die Victoriainsel aufgelockert wird.

Die in der letzten Zeit so leidgeprüfte Metropole des Libanon, bekannt auch unter der Bezeichnung Die Schweiz der Scheichs (Beirut (9) oder "Paris des Nahen Ostens") wird wohl so bald ihre frühere Bedeutung nicht zurückzuerlangen vermögen.

In dem durch seine grossartigen Mäander bekannten, unteren Moseltal entdeckt man die Bernkasteler Schweiz(10).

Die Böhmische Schweiz ist ganz einfach nur die suidliche Fortsetzung des Elbsandstein Gebirges auf dem Territorium der CSSR (11) und weist mit dem Hohen Schneeberg(721m) die höchste Erhebung des gesamten Gebirges auf. Der groteske Formenreichtum der Sächsischen Schweiz wird hier nur im kleinen erreicht.

Die Bremer Schweiz finden wir an der Stelle, 
wo die Lesum kurz vor der Einmündung in die Weser einen rechtsufrigen Steilhang im Geestbereich bildete, der vielfach an die Gegeben heiten der Unterelbe bei Blankenese erinnert, und wo das abwechslungsreiche Gelände landschaftlich besonders anziehende Punkte aufweist, weil dort das Steilufer in "Berg und Tal" aufgelöst wurde. Nach dem Zweiten Weltkrieg musste das Gebiet flächenmässig durch Wohnungsneubauten für die Unterweser-Grossstadt erschlossen werden. Vom raumplanerischen aber auch vom ästhetischen Standpunkt aus gesehen, hat das hügelige und zertalte Hanggelände doch sehr gewonnen (12).

Die Caller Schweiz (Sauerland) zwischen der Kreisstadt Meschede und der teilweise auf einem klassischen Umlaufberg der Ruhr gelegenen Regierungshauptstadt Arnsberg,weist eine stark zertalte Schichtrippenlandschaft auf, (13), die westwärts in den Raum des "Alten Testamentes" überleitet.

Oestlich von Valdivia findet man die Chilenische Schweiz(14), bemerkenswert durch die ausgedehnte Seenplatte; der Lago Llanqui hue ist immerhin halb so gross wie der finnische Saimasee, Imposant wirken die im Osten steil sich erhebenden, teilweise gletscherbedeckten Andengipfel.

Die Daubaer Schweiz bei Duba westlich von Leitmeritz (CSSR) besitzt als formgebendes Element auffallende Quadersandsteinplatten'15). Als ein See- und Moorgebiet kann man die Dümmer Schweiz (16) bezeichnen; der von dem Hunte-Fluss grösstenteils gespeiste Dümmersee ist durchweg recht flach: Grösse rund 15 qkm.

Im Bachtal der zur Ruhr fliessenden Dünnebecke befindet sich etwa seit Beginn unserés Jahrhunderts westlich von Witten eine Gastwirtschaft, die erst einige Jahrzehnte später (etwa 1930) den Namen Dürener Schweiz erhielt. Als Geländebezeichnung soll jedoch der Begriff nie verwandt worden sein(17). Möglich wäre aber durchaus, dass der Volksmund den Ausdruck:"Wir gehen zur Dürener Schweiz"auf den kleinen Talabschnitt übertrug.
Die Elbinger Schweiz, gleichbedeutend mit der Bezeichnung "Elbinger Höhen" $(197 \mathrm{~m})$, ist auch unter dem Namen Dörbecker Schweiz bekannt und ein liebliches Hügelland mit Steilabfall zum Frischen Haff. In beständigem Auf und $\mathrm{Ab}$ wandert man durch wunderschöne Buchenwaldungen, über almartige Wiesen und an Ackerflächen vorüber(18).

Zur Stadtgemarkung Hattingen a. d. Ruhr gehört die Elfringhauser Schweiz, deren Areal etwa dem der Solothurner Exklave Kleinlützel entspricht; man nennt sie auch die Bergische Schweiz. Damit besitzt das Ruhrgebiet seine eigene "Schweiz"; sie wird jetzt(1976) zum grosszügigen Erholungsgebiet ausgebaut. Ihrer Lage zwischen den Städten Essen, Bochum und Wuppertal entsprechend,ist dieses wunderschöne Fleckchen Erde dazu auch prädestiniert. Freizeiteinrichtungen (z. B. Freibad, Spielflächen, Sporteinrichtungen etc. ) sollen nur am Rande dieses Wald-und Wiesen-Idylls entstehen, damit die sogenannte "Ruhezone" mit ihrem ursprünglichen Zustand nicht weiter belastet wird. Wander- und Waldlehrpfade sorgen für ausreichende Erschlies sung (19).

El Salvadorianische Schweiz vgl. Salvadorianische Schweiz. Kein Wunder, dass der Lake District die "Nachprägung" Englische Schweiz (20) erhielt. Und das auch mit vollem Recht, denn die durch die Talvergletscherung geformten Trogtäler verleihen dem Gebirge alpinen Charakter; und was wäre der Lake District ohne die zahlreichen Gewässer, z. B. ohne den Ullswater, den "edelsten der englischen Seen" (21)?

Unweit der Werraquelle kann die Fehrenbacher Schweiz(22) aufgesucht werden, deren nähere und weitere waldreiche Umgebung geologisch sehr instruktiv ist. Bis zum Jahre 1918 zeigte ihre politische Karte die typischen Merkmale der mitteldeutschen Kleinştaaterei, ein Mosaik, das kaum bunter gestaltet werden könnte.

Rund 60 Kilometer nördlich von Helsinki, der Metropole von Finnland, liegt hart westlich der Eisenbahnlinie nach Tampere die Finnische Schweiz(offizieller Name in finnisch:"Sveits $i$ "). 
Es handelt sich dabei um ein relativ kleines Gelände, das zur Stadt Hyvinkää gehört und besonders im Winter für Skiläufer ein beliebtes Ausflugsziel bedeutet. Auf die obligate montägliche Frage nach dem Wochenende erhält man oft zur Antwort:"Olin hiihtämässä Sveitsissä"("Ich war in der Schweiz skilaufen! "Die Region ist als typisches Moränen- und Blockgelände teilweise stark coupiert und ausreichend waldbedeckt. Hotel und Ausflugsrestaurant, Sprungschanze und Kongresshalle, lassen Rückschlüsse auf die steigende Bedeutung dieser kleinen "Sveitsi" zu (23).

Die Fränkische Schweiz beiderseits des Wisentflusses in der Fränkischen Alb (früher: Fränkischer Jura) gehört mit zu den meistbesuchtesten Schweizen, weil man dort noch auf natürlichem Boden steht, auf romantischen Pfaden wandeln und kulturellen Liebhabereien nachzugehen vermag. Dazu kann man lesen: "Die Täler der Fränkischen Schweiz sind noch immer imponierend, die Felsen bizarr und fantastisch, die Höhlen aufregend, die Mühlen melancholisch wie die Burgruinen, die Fachwerkgesichter ernst und bestimmt, die Wälder erfrischend wie die Brunnen und die Menschen" (24). Wer groteske Felsformen studieren will, kommt hier auf seine Kosten; gewisse Aehnlichkeiten mit dem Schweizer Jura, ganz besonders in den Klusen, sind nicht zu leugnen.

Da wo die Gailitz in einem aus Triaskalken bestehenden Tal zwischen Tarvis und Goggau die Fuge (Karnische Alpen/Karawanken) durchbricht, heisst diesés heute durch Wege erschlossene Gelände Gailitzer Schweiz(25).

Die Garbsener Schweiz ist eine $5 \mathrm{~km}$ lange und bis zu $600 \mathrm{~m}$ breite Binnendüne, aus der für den Autobahnbau Dortmund-Hannover soviel Erdreich entnommen werden musste, dass der heute von Mischwald umgebene "Blaue See" entstehen konnte; unweit bildet.ein LeineMäander einen $10 \mathrm{~m}$ hohen Prallhang. In der Saison besteht Schiffsverkehr zwischen Hannover-Linden und dieser mit landschaftlichen Reizpunkten und Gaststätten versehenen "Schweiz"(26).
Als Gütersloher Schweiz gilt die hügelige Dünenlandschaft an der Ems beiderseits der Herzebrocker Strasse an der "Neuen Mühle". Diese Binnendünen entstanden im letzten $\mathrm{Ab}$ schnitt der Eiszeit durch Verwehungen der von den Gletschern hinterlassenen Sandmassen(27).

Angesichts der Porta Westfalica am Durchbruchstal der Weser, entdecken wir die Hausberger Schweiz in einem Kamesgebiet mit Hügeln und Wällen der Grundmoränenlandschaft, herrlichen Moränentäler und Schluchten (28/29).

Im Bereich der Haustenbecker Schweiz (Senne) handelt es sich um ein sehr bewegtes, aus Dünenfeldern und Endmoränenplatten mit tief eingeschnittenen Kastentälern zusammengesetztes Gebiet(30).

Die Hersbrucker Schweiz besitzt viel Aehnlichkeit mit der Fränkischen Schweiz; beide befinden sich nicht weit voneinander entfernt. Die grotesken Felsformen des Pegnitztales und seiner Nebentäler werden alljährlich von vielen Ausflüglern bewundert.

Auch die Hessische Schweiz liegt angesichts des Naturparks Hoher Meissner sehr günstig.

Für den Westen des Himalaya Gebirges ist die Bezeichnung Himalayische Schweiz geprägt worden(31).

Die Hinsbecker Schweiz - auch Kempener Schweiz genannt - umfasst nur den mittleren Teil eines bis zu $80 \mathrm{~m}$ hohen und rd. $20 \mathrm{~km}$ langen plateauartigen, bewaldeten Höhenzuges zwischen der Stadt Viersen und Straelen-Herongen, dessen Entstehung auf erdgeschichtliche Hebungs - und Senkungserscheinungen zurück zuführen ist (32). Dieser Höhenzug stellt im Vergleich zu der flacheren niederrheinischen Landschaft natürlich eine auffallende Erscheinung in diesem Raum und für Erholungsuchende ein attraktives Ziel dar.

Ein zwischen Wurzen und Schildau(Sachsen) gelegener, markant aus seiner Niederungsumgebung herausragender Porphyr-Höhenzug(241m) wurde mit der Bezeichnung Hohburger Schweiz belegt(33). 
Bereits Ende des 18. Jahrhunderts konnte man in einer Beschreibung des Lübecker Malers Heinrich Grosch lesen:"Ich glaube also, dass Holsteins Gegenden ein nicht unwürdiges Pendant zur Schweiz ergeben"(34). Und im Jahre 1885 wurde ein Hotel und ein nahegelegener Bahnhof an der Strecke Malente - Lütjenburg - sie wurde 1976 leider ausser Betrieb gesetzt - Holsteinische Schweiz benannt. Verkehrsmässig liegt sie im Städtedreieck Hamburg Kiel - Lübeck sehr günstig; in der Hauptsaison sind Hotels, Privatpensionen und andere Unterkunftsmöglichkeiten schon lange vor Beginn ausgebucht. Vom Aussichtsturm auf dem Bungsberg(168 m), einem der höchsten Erhebungen des Baltischen Landrückens, geniesst man einen umfassenden Rundblick über die mehr als hundert Seen und grösseren Teiche, die im "Kurpark zwischen den Meeren" von herrlichen Buchenwaldungen und lieblichen Hügeln eingerahmt werden. Gepflegte Städte wiePrestz, Plön, Malente und Eutin,mit bemerkenswerten Bauten bilden beliebte Anziehungspunkte in der Holsteinischen Schweiz(35).

Indische Schweiz (Kaschmir) in Geographenkreisen durchaus geläufig (36).

Auch in Namibia findet sich ein Beispiel:"In den früheren Jahren wurde von der an der Westseite unserer Stadt (Keetmanshop) gelegenen verwitterten Dolorit Kuppengruppe von den deutschsprachigen Einwohnern als von der Keetmanshoper Schweiz gesprochen. Der Grund dafür ist uns unbekannt, da nur bekannt, dass dort nach der Regenzeit, wenn alles grün war, gern Ausflüge abgehalten wurden"(37).

Die Keuchinger Schweiz (38) in unmittelbarer Nachbarschaft des über zwölfhundert Jahre alten Mettlach gelegen, leitet über zum waldreichen Cloefgelände, von wo man den herrlichen Saarschleifenausblick geniesst.

Im Land Hadeln (südöstlich von Cuxhaven)unweit des auffallend aus den Niederungen der Unterelbe herausragenden Geestrückens Wingst $(74 \mathrm{~m})$ und des unter NN liegenden Sintfeldes $(-0,2 \mathrm{~m})$, wo ehedem das Wasser "Feind Nr 1 " war, wird die Klinter Schweiz, im Flussbogen der Oste, für Wassersportler immer mehr ein starker Anziehungspunkt (39).

Etwa südwestlich der anmutig gelegenen Kreisstadt Saarburg (Rheinland-Pfalz) entdeckt man zwischen Freudenburg und Kirf (Saargau) die Kollesleuker Schweiz im Bereich des tief in die Hochfläche eingeschnittenen Leukbaches. Die "Alte Burg", früher zur Abtei St. Maimin in Trier gehörig, beherrrschte ehedem das von Steinhalden und Pilzfelsen, von Buntsandstein und dichtem Mischwald durchsetzte Landschafts bild; in Felsgräben machte man römerzeitliche Funde (38).

Auf der westlichen Abdachung des Westerwaldes hat die Kroppacher Schweiz, welche von den stark mäandrierenden Flüssen (Grosse und Kleine Lister) eindrucksvoll zertalt wird, ihren Standort mit der Giesenhausener Höhe (höchste Erhebung: $360 \mathrm{~m})(40)$. Als Naturs chutzgebiet wird das Gelände immer mehr dem Fremdenverkehr nutzbar gemacht.

Sehr wenig weiss man heute (1977) über das sandige Hügelland der Kurischen Schweiz (Baltikum); wahrscheinlich sind wie in der "Oesel'schen Schweiz" auf dem Agrar- und Forstsektor bedeutende Wandlungen eingetreten.

Vollkommen vom Territorium der Republik Südafrika umgeben, mit bis über $3000 \mathrm{~m}$ kühn aufragenden, almbedeckten Gipfeln ist Lesotho die Schweiz von Afrika. Aufgrund der topographischen Verhältnisse meiden Eisenbahnen und Hauptstrassen das Gebiet, das Flugzeug wurde zum wichtigsten Verbindungsglied mit der Aussenwelt (41).

Die Schweiz des Orients (Libanon) umfasst die höher gelegenen Gebirgsteile, in denen vor dem Krieg der Fremdenverkehr eine zunehmende Rolle spielte; insbesondere war dort der Skisport sehr beliebt.

Für die Lippische Schweiz am Hangstein des Teutoburger Waldes, südlich vom HermannsDenkmal, wird ein Sandsteinzug in Verbindung 
mit den Quellbächen im Cenomanmergelaus raum für das lebhafte Relief (zertalte Schichtrippenlandschaft) bestimmend (42).

Von der Livländischen Schweiz darfman sagen, dass sie an Lieblichkeit kaum zu übertréffen sein wird. Mitten im Baltikum trifft man auf ein richtiges Bergland (Gaizana Kalns $315 \mathrm{~m}$ ), sanfte Hügelgruppen und anmutige Seen, dazu fischreiche Gewässer.

Ganz im Gegensatz zur vorherrschenden Agrarstruktur der dänischen Insel Lolland steht die Lolländische Schweiz bei Vesterborg, eine kleine hügelige, bewaldete Landschaft (43).

Fährt man von Luxemburgs Hauptstadt das Sauertal aufwärts bis Echternach, so erreicht man in dieser geschichtsträchtigen und an Merkwürdigkeiten nicht gerade armen Stadt den Mittelpunkt der Luxemburgischen Schweiz; die Namensprägung soll von Niederländern stammen. Typisch sind romantische Täler mit einem weitverzweigten System von Gründen und Schluchten, wo keine der mitunter grotesken Felspartien einer anderen gleicht; die Luxemburgische Schweiz ist in Richtung Eifel (BRD) grenzüberschreitend (44).

Der Begriff Mährische Schweiz wird nur gelegentlich für den dortigen Karst angewandt(45).

Die westlich vom Oderbruch gelegene Märkische Schweiz (46), auch unter dem Namen Buckower Schweiz bekannt, gruppiert sich um den von Waldungen umgebenen Scharmützelsee, vielen Berlinern nicht unbekannt.

Die im südlichen Ostpreussen anzutreffende Masurische Schweiz, auch als Masurische Seenplatte bezeichnet, weist sehr viele Seen auf, beispielsweise den Spirdingsèe rd. $107 \mathrm{qkm}$ (Zürichsee: $88,5 \mathrm{qkm}$ ); ihren Rahmen bilden ausgedehnte Kiefernwälder und bis zu $160 \mathrm{~m}$ hohe Hügelketten.

Als Mittelpunkt der Mecklenburgischen Schweiz kann ganz zweifellos der Malchinersee in einem waldreichen Gelände bezeichnet werden (47).
Bei der Meinberger Schweiz handelt es sich um eine südöstlich vom gleichnamigen Kurbad befindliche, liebenswerte Talung mit kleinem See und parkähnlichen Anlagen; das alles wird von sanftansteigenden Bergen und wunderschönen Buchenwaldbeständen eingerahmt (48).

In den Abruzzen umspannt die Mittelitalienische Schweiz die Hochmulde von L'Aquila, überragt vom Massiv des Gran Sasso d'Italia(Mte. Corno Grande, $2914 \mathrm{~m}$ ) (49).

Die Moldauische Schweiz. (Eine solche gibt

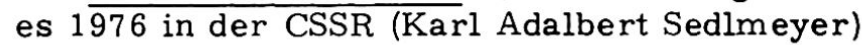
und in Rumänien (Geographisches Institut der Universität Bukarest) nicht. Dazu schreibt jedoch K.-A. Sedlmeyer (1976) wie folgt:"Dort" (Rumänien) "wird das Gebiet um den Cealau (1911m) von Touristen wegen seines Gebirgs charakters" (Oberlauf der Bistrita mit Stausee)" fleissig besucht. Es ist nicht abwegig, dieses Gebiet als 'Moldauische Schweiz' zu bezeichnen. ")

In einer typischen Urstrom-Landschaft liegt die Mosigkauer Schweiz bei Dessau (50).

Weder im mittleren Elbegebiet noch im Naturpark Vogler-Solling konnte die Mühlberger Schweiz ausfindig gemacht werden; das gilt auch für den Raum Gleichen (Bez. Erfurt) mit dem Dorf Mühlberg. (Dazu das Amt der O. ö. Landesregierung in Linz (1976):" . . da ja das Mühlviertel"(mit dem Dorf Mühlberg) "eine sehr charakteristische Landschaft nördlich der Donau ist. Allerdings kennt auch die landeskundliche Literatur für das Mühleviertel keinen Vergleich mit der Schweiz."

Im nordwestlichen Sauerland findet man die Nahmer Schweiz als stilles Wiesental, dessen Steilhänge zumeist bewaldet sind und in die Hochflächen mit Feldbewirtschaftung übergehen.

Die Neuffener Schweiz, südöstlich und unterhalb von Württembergs grösster Burgruine (Hoher Neuffen, $743 \mathrm{~m}$ ) im Bereich der Flurbezeichnung "Schanz", am markanten Steilabfall der Schwäbischen Alb gelegen, ist Teil eines mit vielen 
Tafeln und Schaubildern versehenen geologischen Lehrpfades, der dem Interessierten die erdgeschichtliche Entwicklung der Jurakalke sehr eindrucksvoll vor Augen führt (51). Von allen "Schweizen" befindet sich die am Hohen Neuffen gelegene der Schweizer Grenze am nächsten.

Neuseeland wartet gleich mit drei "Schweizen" auf (52): 1. Die beiden neuseeländischen Inseln unter der Bezeichnung Neuseeland - Schweiz

des Pazifiks, 2. die ganze Südinsel als Schweiz der Südsee und 3. die neuseeländischen Alpen als Neuseeländische Schweiz . Landschaftliche Attraktionen sind zahlreich. Rotorua (Nordinsel) ist das sehenswürdigste Thermalgebiet der Welt; die Kraft der Geiser sowie die kochenden $\mathrm{T}$ eiche und der brodelnde Schlamm bilden einen scharfen Kontrast zu den friedlichen Seen und Wäldern. Im Zentrum der Insel findet man drei wenig aktive Vulkane; der fast perfekt geformte Kegel des Mount Egmont erhebt sich über die üppigen Weiden von Tranaki. Die einmalige Schönheit der Southern Alps, (Südinsel), die von dem $3760 \mathrm{~m}$ hohen Mt. Cook (Gr. Glockner: $3797 \mathrm{~m}$ ) gekrönt werden, ist wohl bekannt. Die Seen Te Anau und Manpouri, die schönsten Seen des Landes, bilden das Tor zum Milford Sound, ein eiszeitliches, heute meererfulltes Gletschertal. Andernorts an der Westküste der Suldinsel reichen die Eismássen der Fox-und Franz-Josef-Gletscher auch heute noch bis zum Meer, so dass dieses fjordartige Gestade durchaus "Alaska der Südsee"heissen könnte(53)!

Die Schweiz der Niederlande scheint wohl als Begriff in Geographenkreisen bekannt zu sein, doch war leider der genaue Standort nicht zu ermitteln. (AmbassadeRoyale Des Pays-Bas (Bonn), 1976: ". . . keine Schweiz der Niederlande bekannt".)

Keine natürliche Landschaftsform, sondern ein künstlich geschaffenes Gebilde und somit ein Kuriosum, ist die mitten in Köln gelegene Nippeser Schweiz. Sie war früher eine kleine baum - und strauchbestandene Parkanlage zwischen einem Gleisdreieck, die in einer Schlucht (alter Rheinarm) gelegen war. Nach dem letz- ten Weltkriege wurde alles mit Trümmerschutt aufgefüllt und darüber hinaus ein "Gebirge" erstellt, eine ein Kilometer lange Hügelkette geschaffen und alles einer neuen Parkanlage angeglichen (54).

Südlich von Caen deckt sich flächenmässig La Suisse Normande etwa mit dem in der westlichen Normandie befindlichen Landschaftsteil "Bocage Normand"; sie hat ihre "Prägung" deshalb erhalten, weil die an und für sich ebene Normandie gerade dort etwas hügelig ist und viel Milchwirtschaft betrieben wird, also wenigstens einige Anklänge an die Schweiz bietet. "Les routes de Caen a Domfront et celle de Caen a Mortain desservent les plus beaux sites et les principales localités d'une des régions naturelles les plus caractéristiques de la Normandie, à qui son relief accidenté, ses gorges profondes, ses eaux courantes, ses paturages et ses forets ont valu l'appelations de "Petite Suisse" ou "Suisse Normande". C'est un surnom trop ambitieux, et plutot fait pour desservin ce pays pourtant si pittoresque. Combien lui convient mieux son nom géographique de "Bocage", qui exprime parfaitement a lui seul la nature du sol et les accidents de son relief, le caractere de sa végétation et l'aspect de ses paysages (55)."

Wenn man sich vom Bad Oeynhausener Kurpark auf der Bismarckstrasse ostwärts wendet, wird nach ganz kurzer Entfernung eine kleine 13 Hektaren Fläche umfassende Grünanlage erreich für die der Name Oeynhausener Schweiz geprägt wurde, die allen Einheimischen ein Begriff ist (56

Ganz im Süden des ehemaligen Grossherzogtums und heutigen Verwaltungsbezirks Oldenburg kann die Oldenburgische Schweiz (57) besucht werden; es handelt sich dabei um ein Hügelland $(146 \mathrm{~m})$, das au ch vielfach "Dammer Schweiz" genannt wird.

Die Oesel'sche Schweiz auf der gleichnamigen Insel (estnisch:Saaremas mit 2714 qkm Fläche; Gotland $3140 \mathrm{qkm}$ ), wo vonaltersher Landwirt schaft, Viehzucht und Fischfang betrieben werden, besitzt ein durch Waldungen, Felder und Wiesen abwechslungsreich gestaltetes Land- 
schaftsbild, das durch zu Gaststätten ausgebaute Windmühlen angenehm belebt wird (58).

Die Diemeltalsperre, welche für die Regulierung des Weserwasserstandes sehr wichtig ist, liegt in der Padberger Schweiz und gewinnt damit an Anziehungskraft.

Im Mittelpunkt der Panamenischen Schweiz steht an der Westgrenze von Panama der Chiriqui Vulkan (Volcan de Chiriqui: $3478 \mathrm{~m}$; Aetna $3263 \mathrm{~m})$, der als erloschen zu betrachten ist (59).

Die Klein Schweiz im Penninischen Gebirge ist einer breiten Oeffentlichkeit durch ein schweres Verkehrsunglück, wobei 32 Reisende ums Leben kamen, in Erinnerung gebracht worden. Ein Autobus kam auf abschüssiger Strassenstrecke vom Wege ab und stürzte in eine der für diesen Gebirgsteil so typischen Schluchten (60).

Nahe bei Neumarkt (Obersteiermark) hat der Perchauerbach im Serizit-Quarzphyllit eine epigenetische Schlucht mit rund $20 \mathrm{~m}$ hohen Felshängen geschaffen. Die Bezeichnung Perchauer Schweiz stammt aus der Zeit (1880), als

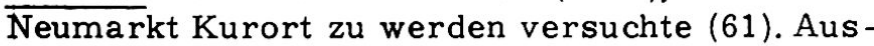
gerechnet durch diese Schlucht musste eine Bundesstrasse trassiert werden, womit viel von den landschaftlichen Reizen verloren ging!

Mit drei verschiedenen "Nachprägungen" vermag die folgende der "Schweizen" aufzuwarten:Die Polnische Schweiz (62), auch Kaschubische Schweiz (Szwajcaria Kaszubska) oder Karthauser Schweiz, beherrscht vom $330 \mathrm{~m}$ hohen Turmberg. Fast unmerklich geht südwestwärts das gewässerreiche Gebiet in die Pommersche Schweiz über; diese ist ein Teil des Pommerschen Landrückens, der rund 2000 Seen (z. B. Madúsee 36, $8 \mathrm{qkm} /$ Brienzersee 29, 0qkm/ aufweist (63).

Die Pönitzer Schweiz (64) unweit der holsteinischen könnte infolge des Seenreichtums als deren kleiner Ableger bezeichnet werden.

Südöstlich von Bad Kreuznach liegt die RheinHessische Schweiz.
Eine dichtbewaldete, mit vielen Felsbrocken übersäte und verwilderte Region in den Karawanken,ist die Rosentaler Schweiz, die heute durch Anlagen von Wanderwegen als Erholungsgebiet erschlossen werden soll (65).

Das kuppige Gelände der Rüdigsdorfer Schweiz. nördlich von Nordhausen ist an die ZechsteinGipsberge des Südharzes im Umkreis von Rüdigsdorf gebunden (66).

Im Verwaltungsbereich des Kreises Holzminden-Land befindet sich die durch ihre eindrucksvolle Kirschblütenzeit bekannte Rühler Schweiz, ein dem Vogler-Massiv (Weserbergland)vorgelagertes Hügelgelände im Bereich der Ortschaften Bodenwerder, Rühle und Golmbach (67).

Die Ruppinsche Schweiz, nordwestlich von Berlin, wird wegen ihres Gewässerreichtums viel besucht.

Zwischen Mettlach und Saarhölzbach soll die Saarländische Schweiz erwähnt werden:grosse Waldbestände und Zeugen von hohem kulturhistorischem Wert, beispielsweise die Burgruine Montclair, prägen hier die Landschaft.

Auf die Arbeit von Alfred Hettner über die Sächsische Schweiz (69), wurde bereits hingewiesen; es handelt sich hierbei zweifellos um die eingehendste Bearbeitung einer der vielen "Schweiz-Regionen": bastionförmige Erhebungen (Lilienstein) und seltene Felsbildungen (Gr. Winterberg), klammartige Talungen(Nebentäler) und sehenswerte Aushöhlungen (Kuhstall); das ganze Elbsandsteingebirge ist durchsetzt von grotesken Formen.

Der Begriff Salvadorianische Schweiz wird gelegentlich gesprächsweise für die Berglandschaft in der Nähe der Provinzhauptstadt La Palma verwendet; dies jedoch wohl ausschliesslich von deutschsprachigen Einwohnern (70).

Grosse landschaftliche Uebereinstimmungen bestehen zwischen der Oesterreichischen Schweiz (Salzkammergut) und der Schweiz selbst; diese "Nachprägung" ist leicht verständlich 
Um den Telezker See (230 qkm, Lago Maggiore: 216 qkm) gruppiert sich die Sibirische Schweiz (71) in einem geradezu beneidenswert schönen, zum Altaigebirge gehörenden Landschafts raum, der zum Erholungsgebiet prädestiniert ist.

Der Ausdruck die Spanische Schweiz wurde gleich für zwei Gebiete verwendet: die Costa Blanca, immer mehr als Feriengebiet bevorzugt, und Almeria (72) am Südhang der eindrucksvollen Sierra Nevada.

Gewässer-Reichtum, Viehweiden und bewaldete Hügelketten zeichnen die Stormarnsche Schweiz (73); damit besitzt Holstein drei "Schweizen".

Die Süditalienische Schweiz deckt sich etwa mit dem Sila-Gebirge (1930 m) in Kalabrien. Waldungen, Seen, Weiden und Fruchtbaumhaine sind typisch; ein Stausee wurde für das Silawerk angelegt. Zunehmender Fremdenverkehr ist $\mathrm{zu}$ verzeichnen (74).

Im nördlichen Pakistan liegt im Gebiet von Swat eine Hochgebirgsregion, die zu den "Schweizen" gehört. Unweit der Verwaltungshauptstadt Saidu kann man über eine kühn angelegte Strasse inmitten von Aprikosen- und Olivenhainen den Sommer-Erholungsort Marghzar in einem einsam gelegenen Tal erreichen; dichter Waldbestand mit kristallklaren Gewässern erhöht noch die Entrücktheit. Die einfachen Hütten von Madyan (1550 m) ducken sich verwegen an den Steilhängen des eigentlichen Swattales. Anbau ist nur möglich, weil Steinwălle gegen die Witterungsunbilden Schutz gewăhren. Dort, wo auf einem Hochplateau der $\mathrm{Zu}$ sammenfluss zweier Ströme den Swatriver bildet, liegt Kalam, mit Recht als Höhepunkt der Swatlandschaft bezeichnet. Das westwärts gelegene Gabratal konnte bis auf den heutigen Tag unberührte Wildheit und romantische Schönheit erhalten:Die Schweiz des Ostens (75).

Törtensche Schweiz im Urstromtal bei Dessau (76).

Die engen Durchbruchstäler der Weissen Elster und des Göltzsch-Flusses waren wohl der Grund, in diesem landschaftlich so reizvollen Gebiet von einer Vogtländischen Schweiz zu sprechen. (77).

Eine der grösstenzusammenhängenden Waldungen Mitteleuropas im Privatbesitz dehnt sich östlich von Bad Berleburg im Hochsauerland aus und wird von der $816 \mathrm{~m}$ hohen Ziegenhelle überragt: Die Wittgensteiner Schweiz, wo heute noch stundenlange Wanderungen möglich sind, ohne irgendjemandem zu begegnen.

Die Wolkensteiner Schweiz liegt im westlichen Teil der Nordabdachung des Erzgebirges, wo das Relief sehr bewegt ist. Ueber einem tief eingeschnittenen $\mathrm{Tal}$ hat die gleichnamige Burg auf einem Felsvorsprung ihren Standort; das ganze "Schweizgebiet" ist von dort einzusehen.

Abschliessend könnte man natürlich auch fragen, ob nicht auch die Schweiz fremde Länderbezeichnungen "entlehnt" hat. Dies ist da und dort, doch meist scherzhaft der Fall gewesen: Val Calanca = Kalabrien der Schweiz, Wallis= Kalifornien der Schweiz, Zollikon = Beverly Hills der Schweiz (78), usw., wären dafür Beispiele. Doch kommen auch Ortsbezeichnungen wie Algerien, Helgoland usw. vor, die andern Ursprungs sind.

\section{Quellenangaben:}

( 1 ) Alfred Hettner:Gebirgsbau und Oberflächen gestaltung der Sächsischen Schweiz. In:Forschungen zur Landeskunde, Bd. 2, 1887, S. 249 355.

( 2) Irmfried Siedentop: Der "Schweiz"-Begriff auf andere Landschaften übertragen. In: Zeitschrift für Wirtschaftsgeographie, 1973, S. 9-12. Ders. :"Schweizen"in aller Welt. In: Neue Zürcher Zeitung, 1975, Nr. 299, S. 37-38 . ( 3 ) Anton Sterzl und Emil Bauer:Fränkische Schweiz. Bamberg 1976.

( 4) Fr.Slezak(Wien):Briefliche Mitteilung 1973.

( 5) Wolfgang Senftleben (Taipeh):Briefliche Mitteilung 1973.

(6) Irmfried Siedentop, 1975, a. a. O.

( 7) Bernhard Hopf(Bern):Briefliche Mitteilung 1976. 
( 8) Ambassade de Suisse en Argentine (Buenos Aires): Briefliche Mitteilung 1976 und Unterlagen der Argentinischen Botschaft (Bonn) 1973.

(9) Westfalenpost (Hagen i. W. )1976.

(10) Ernst Winkler (Zürich):Privatsammlung 1973.

(11) Karl Adalbert Sedlmeyer (München): Briefliche Mitteilung 1973.

(12) Schulwirklichkeit und Erziehungswissenschaft, Bremen 1967, S. 182.

(13) Ludwig Maasjost (Paderborn):Privatsammlung 1976.

(14) Brockhaus Enzyklopädie. Bd. 3, 1967, S. 757.

(15) Karl Adalbert Sedlmeyer, a. a. O.

(16) Martin Schwind(Hannover):Mündliche Mitteilung 1976.

(17) Wilhelm Nettmann (Witten a.d. Ruhr): Briefliche Mitteilung 1975.

(18) Bruno Hoffmann und Theodor Hurtig: Ostpreussen, Land und Leute. Königsberg o. J. (19) Stadtverwaltung Hattingen a.d. Ruhr: Unterlagen 1976.

(20) Karl Eckart(Neuss):Briefliche Mitteilung 1975.

(21) Peter Fischer:Grossbritannien und Ir land. Frankfurt a. M. 1976.

(22) Ernst R. Fugmann(Hamburg):Briefliche Mitteilung 1976.

(23) Peter Neuenschwander(Helsinki): Brieflịche Mitteilung 1976.

(24) Anton Sterzl und Emil Bauer, a. a. O.

(25) Herbert Paschinger (Graz):Briefliche Mitteilung 1976.

(26) Martin Schwind (Hannover):Briefliche Mitteilung 1976.

(27) Werner Lenz (Gütersloh):Briefliche Mitteilung 1973.

(28) Diethelm Düsterloh (Bielefeld):Privatsammlung.

(29) Hausberge an der Porta in Gegenwart und Vergangenheit $\mathrm{o}$. J.

(30) Diethelm Düsterloh, a. a. O.

(31) Embassy of India (Bonn):Briefliche Mitteilung 1976.

(32) Zweckverband Naturpark Schwalm-Nette (Kempen): Briefliche Mitteilung 1976.

(33) Martin Schwind, a. a. O.

(34) Anton Sterzl und Emil Bauer, a.a. O.
(35) Irmfried Siedentop 1975, a. a. O. (36) z. B. Albert Leemann(Zürich):Briefliche Mitteilung 1976.

(37) Municipality Keetmanshoop (Namibia): Briefliche Mitteilung 1976.

(38) Unterlagen der Stadt Mettlach und des Verkehrsamtes Saarburg 1976.

(39) Rudolf Lembcke:"Kreis Land Hadeln". Otterndorf(Niederelbe) 1976.

(40) Adolf Hövels:"Die Kroppacher Schweiz". Kroppach (Westerwald) o. J.

(41) Der Neue Tag. Weiden(Oberpfalz) 1976.

(42) Ludwig Maasjost,a.a. O.

(43) Baedekers Skandinavien. Stuttgart $1972 / 73$, S. 82.

(44) Echternach und Umgebung, die Luxemburgische Schweiz. Luxemburg 1956. (Vermittelt von Werner Kreuer, Essen).

(45) Karl Adalbert Sedlmeyer, a. a. O.

(46) Ernst Winkler,a.a. O.

(47) Gerhard Böhmer: Die eigenen Landschaftswerte der Mecklenburgischen Schweiz. In: Geographische Wochenschrift 1934, S. 234237.

(48) Unterlagen der Kurverwaltung Bad Meinberg 1976.

(49) Anton Sterzl(Köln):Briefliche Mitteilung 1976.

(50) Wolf Donner (Port-au-Prince):Briefliche Mitteilung.

(51) Stadtverwaltung Neuffen:Unterlagen(Albert Allgaier:Auf dem Ammoniten-Pfad ins Erdmittelalter o. J.).

(52) Hildesuse Gaertner:Neuseeland - kein Paradies ohne Schatten. In:Zeitschrift für Wirtschaftsgeographie, 1976, S. 221 -223. Dies. (Freiburg i. Br. ):Briefliche Mitteilung 1976.

(53) Wissenswertes über Neuseeland (New Zealand Government Tourist and Publicity Department), Wellington 0 . J.

(54) Wolfgang Ute(Kassel):Briefliche Mit teilung 1976 .

(55) Französiche Botschaft (Bonn):Briefliche IMitteilung 1976; ferner Ablichtung aus: Guide Bleu, Normandie (48. - DE CAEN A MAYENNE ET DE MAYENNE A CAEN) O. J.

(56) Vgl. Prospekte der Kurverwaltung Bad Oeynhausen o. J. 
(57) Ernst Winkler, a. a. O.

(58) Wladimir Weiman:Neues Leben auf

Saaremas. In:Sowjetunion - heute, 16/1976.

(59) Ernst Winkler, a. a. O.

(60) Westfalenpost (Hagen i. W. ) 1975.

(61) HerbertPaschinger, a. a. O.

(62) Geographisches Institut der Universität

Krakau: Briefliche Mitteilung 1976.

(63) Brockhaus Enzyklopädie. Bd. 14, 1972, S. 794.

(64) Gustav Peters. In:Blätter für Heimatkunde, Eutin 1974, S. 82.

(65) Gemeindeamt St. Jakob, Bezirk Villach: Briefliche Mitteilung 1976.

(66) Oskar August (Halle a. d. S. ):Briefliche Mitteilung 1976.

(67) Stadt Holzminden (Fremden- und Kulturamt):Briefliche Mitteilung 1976.

\section{Literaturbesprechungen}

SPAKRS, B. W. und WEST, R. G. : The Ice Age in Britain; 302 S. , 127 Fig. , 44 Photos, Methuen \& Co Ltd London, 1972, £5. 50.

Dieses Buch versteht sich als Informations quelle und Orientierungshilfe sowohl für Studienanfänger der naturwissenschaftlichen Disziplinen (Erdwissenschaften, Biologie) als auch für interessierte Laien. Auf leicht verständliche Weise werden die Erscheinungsbilder der glazialen Formenwelt in den ersten Kapiteln behandelt. Auch wird versucht, die Gründe der Eiszeitenentstehung darzulegen, wobei die Autoren die Schwierigkeiten bei der Gewichtung und dem Zusammenwirken der einzelnen Fakten nicht verschweigen. Die erarbeiteten Begriffe werden in den folgenden Kapiteln am Relief der britischen Inselwelt geprüft und eingeordnet. Dabei kommen auch Fragen über das Periglazial und die Stratigra-
(68) Der Chef der Staatskanzlei SAARLAND: Briefliche Mitteilung 1976.

(69) Alfred Hettner, a. a. O.

(70) Embajada de la Republica Federal de Alemania (San Salvador): Briefliche Mitteilung 1976.

(71/72) Ernst Winkler,a.a. O.

(73) Kreisverwaltung Bad Oldesloe:Briefliche Mitteilung 1974.

(74) Anton Sterzl, a. a. O.

(75) M. U. Stefan Malik und Annemarie

Schimmel: Pakistan, Basel 1976

(76) Wolf Donner, a. a. O.

(77) Brockhaus Enzyklopädie. Bd. 19, 1974, S. 679 .

(78) Fr. Slezak, a.a. O.

phie der glazialen und postglazialen Ablagerungen, über die Botanik und Zoologie, aber auch über den Menschen des Eiszeitalters eingehend zur Sprache. Ein weiteres Kapitel widmet sich den Möglichkeiten und Problemen der relativen und absoluten Eiszeitchronologie und den dazu zur Verfügung stehenden Datierungsmethoden. Das vorliegende Werk ist kein Lehrbuch, bietet aber dem Studienanfänger und auch dem interessierten Laien eine gute und klare Einführung in das Gebiet der modernen Eiszeitforschung. Durch die klare Darlegung der allgemeinen Gegebenheiten glazialer Erscheinungen ergeben sich Erkenntnisse und werden Methoden ersichtlich, die auch ausserhalb Britanniens ihre Gültigkeit beibehalten. Gerade deshalb vermag das Buch dem Neuling auf diesem Gebiet manchen wertvollen Hinweis zu geben.

Waldemar A. Keller 